\title{
Factibilidad de integrar split-drain MAGFETs con alta sensibilidad en tecnología CMOS
}

\section{Feasibility to integrate high-sensitivity split-drain MAGFETs in CMOS technology}

\author{
Gerard Franz Santillán Quiñonez ${ }^{1,2}$, Víctor H. Champac Vilela² y Roberto S. Murphy Arteaga² \\ ${ }^{1}$ Departamento de Engenharia Elétrica, Universidade Federal de Santa Catarina, Campus Universitário- \\ Trindade, Florianópolis, Santa Catarina, Brasil, C.P. 88040900, e-mail: gsantil@ieee.org \\ ${ }^{2}$ Departamento de Electrónica, Instituto Nacional de Astrofísica, Óptica y Electrónica, Sta. Ma. Tonantzintla, \\ Puebla, México, C.P. 72840.
}

\section{RESUMEN}

La factibilidad de un Split-Drain MAGFET como sensor magnético ha sido explorada con diversas metodologías, pero sin aprovechar más de un efecto galvanomagnético simultáneamente. Unificando trabajos realizados teórica y experimentalmente, modelos analíticos continuos para la relación entre las fuerzas actuando en la dirección de deflexión y el ángulo de Hall, así como criterios de diseño para incrementar la sensibilidad de un Split-Drain MAGFET son presentados. El análisis propuesto muestra que es posible aprovechar los efectos de deflexión de las líneas de corriente y de magnetorresistencia para incrementar la sensibilidad en un Split-Drain MAGFET. Con un Split-Drain MAGFET con canal considerado como plato de Hall corto, sensibilidades de hasta $59 \% / T$ han sido obtenidas experimentalmente midiendo densidades de flujo magnético desde $90 \mu T$ hasta $275 \mu T$. Esto es posible debido a la contribución de los dos efectos galvanomagnéticos considerados. Adicionalmente, un macro modelo SPICE para un Split-Drain MAGFET es propuesto para facilitar su uso en circuitos de mayor complejidad. Con respecto a los resultados experimentales obtenidos, el macro modelo SPICE propuesto tiene un error $<1.6 \%$ generando el desbalance entre las corrientes de drenaje. Como un Split-Drain MAGFET es compatible con tecnología CMOS, dominante en circuitos integrados, los resultados obtenidos muestran que es factible usarlo como sensor magnético en sistemas integrados CMOS de alta complejidad, lo cual puede abrir un amplio rango de aplicaciones con bajo costo.

Descriptores: MAGFET, split-drain MAGFET, efectos galvanomagnéticos, efecto Hall, magnetorresistencia, sensor magnético.

\section{ABSTRACT}

The feasibility of a Split-Drain MAGFET as magnetic sensor has been explored with several methodologies, but without simultaneously advantaging more than one galvanomagnetic effect. Unifying theorically and experimentally developed works, continuous analytical models for the relationship between forces acting in the deflection direction and for the Hall angle, as well as design criteria to increase the sensitivity of a Split-Drain MAGFET are presented. The proposed analysis shows that it is possible to take advantage of the current-lines deflection and magnetoresistance effects in order to increase the sensitivity of a Split-Drain MAGFET. With a Split-Drain MAGFET with a channel considered as a short Hall plate, sensitivities up to $59 \% / T$ have been experimentally obtained measuring magnetic flux densities from $90 \mu T$ to $275 \mu T$. This is possible due to the contribution of the two considered galvanomagnetic effects. Additionally, a SPICE macro-model for a SplitDrain MAGFET is proposed to facilitate its use in more complex circuits. With respect to the obtained experimental results, the proposed SPICE macro model has an error $<1.6 \%$ generating the drain current imbalance. Since a Split-Drain MAGFET is compatible with CMOS technology, dominating in integrated circuits, the obtained results show that it is feasible to use it as magnetic sensor in CMOS integrated systems of high complexity, which opens a wide range of low cost applications.

Keywords: MAGFET, split-drain MAGFET, galvanomagnetic effects, Hall effect, magnetoresistance, magnetic sensor. 


\section{INTRODUCCIÓN}

Un Transistor de Efecto Campo MAGnético (MAGFET, por sus siglas en inglés) es un transductor que convierte una densidad de flujo magnético en una señal eléctrica. Dos tipos son posibles: a) un Hall MAGFET, que genera un voltaje proporcional a la DFM, o b) un Split-Drain MAGFET (SD-MAGFET) que produce un desbalance entre sus corrientes de drenaje proporcional a la densidad de flujo magnético. Un SD-MAGFET es logrado con un transistor MOS dividiendo el drenaje en varios nodos [1, 2].

El efecto Hall y los efectos de deflexión de las líneas de corriente y de magnetorresistencia son los principales efectos galvanomagnéticos que ocurren en un SD-MAGFET. Los dos últimos pueden influir significativamente en su sensibilidad. Sin embargo, los trabajos desarrollados por otros autores están enfocados principalmente en el efecto de deflexión de las líneas de corriente [2-8]. Con este enfoque, con razón de aspecto equivalente $L_{e q} W_{e q} \geq 1$ y operando en inversión fuerte, las sensibilidades reportadas fueron $<15 \% / T$ [2-8]. La importancia del efecto de magnetorresistencia en platos de Hall con contacto partido (split) fue analizada en [9, 10], mostrando aceptable linealidad con la DFM. Adicionalmente, Dobrovolsky et al. [11] mostraron que el efecto de magnetorresistencia es más importante en la región de saturación que en la región lineal. A pesar de estos factores, otros autores no han reportado una metodología para aprovechar simultáneamente estos dos efectos, lo cual es abordado en este trabajo.

Por otro lado, aún no ha sido reportado un modelo SPICE para un SD-MAGFET convencionalmente aceptado que facilite su inclusión en circuitos integrados. La fuerza de Lorentz actuando sobre los portadores de carga en el canal produce una diferencia de concentración de portadores $\left(\Delta n_{c h}\right)$ entre los lados del canal asociados a cada drenaje. Esto conduce a tener una diferencia entre las conductancias $\left(\Delta g_{d s}\right)$ y el voltaje umbral $\left(\Delta V_{T}\right)$ asociados a estos mismos lados. El modelo SPICE reportado en [12] incluye estas variaciones pero puede ser usado sólo para un modelo específico del transistor MOS (NonQuasi-Static). Adicionalmente, requiere la estimación de un parámetro, $a$, obtenido con un simulador de dispositivos. Macro modelos SPICE para SDMAGFETs reportados [5,6,13] generan algebraicamente el desbalance entre las corrientes de drenaje sin considerar los fenómenos de $\Delta n_{c h}, \Delta g_{d s}$ y $\Delta V_{T}$. Este trabajo propone un macro modelo SPICE para un SD-MAGFET válido para cualquier modelo de transistor MOS que considera los fenómenos de $\Delta n_{c h}, \Delta g_{d s}$ y $\Delta V_{T}$

\section{SPLIT-DRAIN MAGFET: FUNDAMENTOS}

Dividiendo el drenaje de un transistor MOS en dos o más nodos, es posible formar un SD-MAGFET (ver Figura 1). Inmerso en una densidad de flujo de magnético, $\vec{B}_{Z}$, perpendicular al plano de su canal, por acción de la fuerza de Lorentz, un desbalance entre las corrientes de drenaje, $\boldsymbol{I}_{D 2} \boldsymbol{I}_{\boldsymbol{D} 1}$, es generado. $\boldsymbol{I}_{D 1} \mathrm{e}$ $\boldsymbol{I}_{D 2}$ son las corrientes en el drenaje 1 y 2 , respectivamente. Entonces, su sensibilidad, $S$, es definida por:

$$
S=\frac{\Delta I_{D}}{\left(I_{D 1}+I_{D 2}\right) \cdot B_{z}}=\frac{\left|I_{D 2}-I_{D 1}\right|}{\left(I_{D 1}+I_{D 2}\right) \cdot B_{z}}
$$

El canal de un SD-MAGFET funciona como un plato de Hall donde ocurren fenómenos galvanomagnéti-

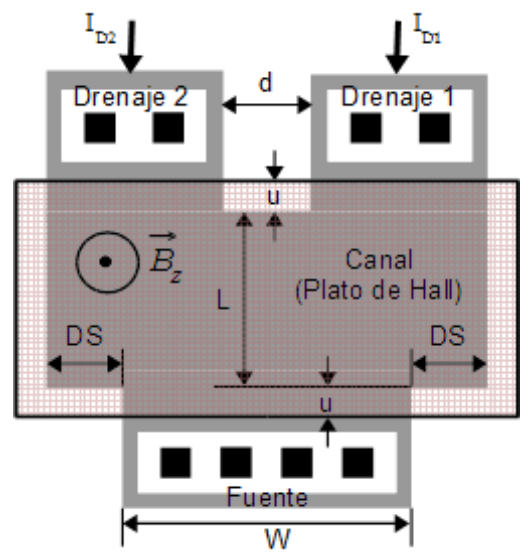

Figura 1: Split-Drain MAGFET con dos drenajes.

cos (ver Figura 2). La acumulación de portadores hacia uno de los lados del canal crea un voltaje de Hall entre cada par de puntos simétricamente ubicados, $M$ y $N$, a lo largo de un plato, lo cual es conocido como efecto Hall. Las líneas de corriente modifican su trayectoria longitudinal generando, con ello, los efectos de deflexión de las líneas de corriente y magnetorresistencia [1, 2].

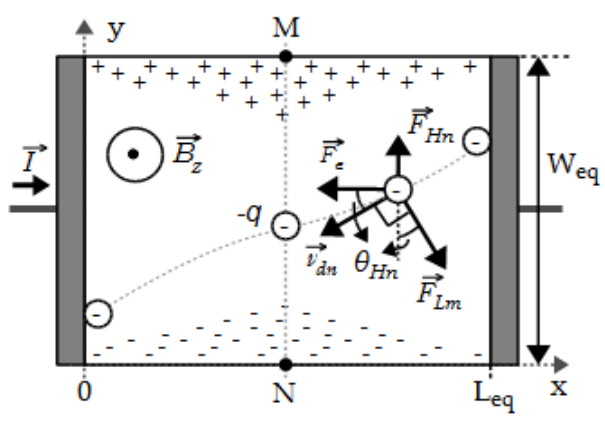

Figura 2: Plato de Hall rectangular tipo $n$. 
ECIPERÚ

Sobre cada portador de carga con una velocidad de arrastre $\vec{v}_{d n}$, actúan la fuerza eléctrica generada por la polarización, $\vec{F}_{e}$, la fuerza eléctrica de Hall, $\vec{F}_{H n}$, y la fuerza de Lorentz, $\vec{F}_{L m}$ (ver Figura 2 y Ecuación 2). El ángulo formado entre la densidad de corriente eléctrica, $\vec{J}_{n B}$, y la dirección longitudinal es llamado ángulo de Hall, $\theta_{H n}$, el cual varía a lo largo del canal. En un plato de Hall tipo $n, \vec{J}_{n B}$ tiene la misma dirección pero el sentido opuesto de $\vec{v}_{d n}$ (ver Figura 2).

$$
\vec{F}_{L m}=-q\left(\vec{v}_{d n} \times \vec{B}_{Z}\right)
$$

\section{FUNDAMENTOS PARA MEJORAR LA SENSIBILIDAD EN UN SPLIT-DRAIN MAGFET}

Para incrementar la sensibilidad de un SD-MAGFET, el desbalance entre las corrientes de drenaje debe ser incrementado (ver Ecuación 1). Para lograr esto, los efectos de deflexión de las líneas de corriente y de magnetorresistencia deben ser dominantes con respecto al efecto Hall. Esto puede ser analizado de a través de la razón entre el módulo de las fuerzas que actúan en la dirección de deflexión y del ángulo de Hall, $\theta_{H n}$ [14-17]. Considerando que existen diversas geometrías para el canal de un SD-MAGFET [2-8], una forma rectangular equivalente es propuesta para mantener generalidad en el análisis $[10,15$, 16] (ver Figura 2).

\section{Razón Entre las Fuerzas que Actúan en la Dirección de Deflexión}

En la dirección de deflexión, las fuerzas que actúan sobre el portador son la fuerza eléctrica de Hall, $\vec{F}_{H n}$, y la componente en esta dirección de la fuerza de Lorentz, $\vec{F}_{L m y}$. Usando la definición del factor de corrección geométrica, $G$, es posible modelar apropiadamente la razón $F_{H n} / F_{L m y}$. El factor $G$ es definido como la razón entre el voltaje de Hall generado en un plato de Hall real, $V_{H n}(x)$, y el máximo voltaje de Hall posible en un plato de Hall ideal, $V_{H_{\infty}}$. Usando relaciones físicas apropiadas para un plato de Hall rectangular equivalente (ver Figura 2), es posible definir:

$$
G(x)=\frac{V_{H n}(x)}{V_{H \infty}}=\frac{W_{e q} \cdot F_{H n} /(-q)}{W_{e q} \cdot F_{L m y} /(-q)}=\frac{F_{H n}}{F_{L m y}}
$$

\section{Modelo del Ángulo de Hall}

Considerando la Figura 2 y la Ecuación 3, es posible obtener:

$$
\tan \theta_{H n}=\frac{F_{L m y}-F_{H n}}{F_{e}}=(1-G(x)) \frac{F_{L m y}}{F_{e}}
$$

De acuerdo a la Ecuación 2 y la Figura 2, $F_{L m y}$ puede ser estimado como $F_{L m y}=-q \cdot v_{d n} \cdot B_{Z} \cdot \cos \theta_{H n}$. Además, $F_{e}=-q \cdot E_{e} \quad$ y $E_{e}=-v_{d n} \cdot \cos \theta_{H n} / \mu_{H n}$, donde $E_{e}$ es el módulo del campo eléctrico por polarización, y $\mu_{\mathrm{Hn}}=r_{\mathrm{Hn}} \mu_{n}$ es la movilidad de Hall. $r_{\mathrm{Hn}}$ es el factor de dispersión de Hall $[1,2]$, y $\mu_{n}$ es la movilidad del portador de carga. Usando las relaciones dadas, uno puede obtener:

$$
\tan \theta_{H n}=(1-G(x)) \mu_{H n} \cdot B_{Z}
$$

El modelo propuesto permite tener una variación continua del ángulo de Hall si es usado un modelo continuo de $G(x)$ [4]. Esto es un aporte sustancial con respecto al valor constante reportado en la literatura, el cual, según el modelo propuesto, es el valor máximo del ángulo de Hall en un plato de Hall, $\theta_{H n^{+}}=\mu_{H n} \cdot B_{Z}$.

\section{Impacto de la Geometría}

Usando las Ecuaciones (3) y (5) y el modelo continuo para $G(x)$ propuesto en [4], el impacto de la geometría del canal puede ser analizado. La variación de $F_{H n} / F_{L m y}$ a lo largo de un plato de Hall corresponde a las curvas de $G(x)$ dadas en [4], donde el valor de $F_{H n} / F_{L m y}$ aumenta cuando la razón $L_{e d} / W_{e q}$ aumenta. Por otro lado, la variación continua del ángulo de Hall normalizado con respecto a su valor máximo es mostrada en la Figura 3. Contrario a lo que ocurre con $F_{H n} / F_{L m y}$, la razón $\theta_{H n} / \theta_{H n^{+}}$aumenta cuando la razón $L_{e q} W_{\text {eq }}$ disminuye. 


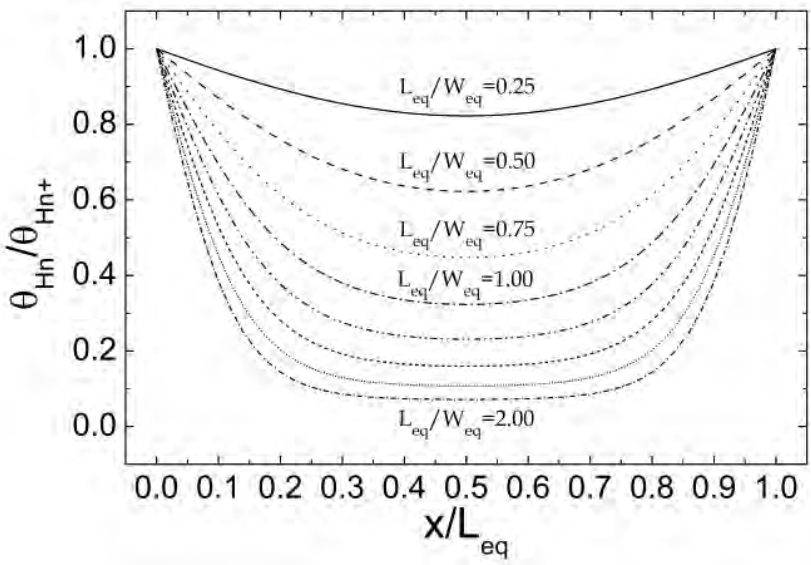

Figura 3: Variación del ángulo de Hall a lo largo de un plato de Hall.

Para determinar los valores adecuados de la razón $L_{e d} W_{e q}$ con los cuales algunos efectos galvanomagnéticos son dominantes, es apropiado analizar los valores de $F_{H n} / F_{L m y}$ y $\theta_{H n} / \theta_{H n^{+}}$en $x=0.5 \cdot L_{e q}$. En este punto del canal, de acuerdo al modelo usado de $G(x)$, el valor de $F_{H n} / F_{L m y}$ es máximo y el de $\theta_{H n} / \theta_{H n+}$ es mínimo. La Figura (4) muestra la variación de ambas razones con $L_{e q} W_{\text {eq }}$.

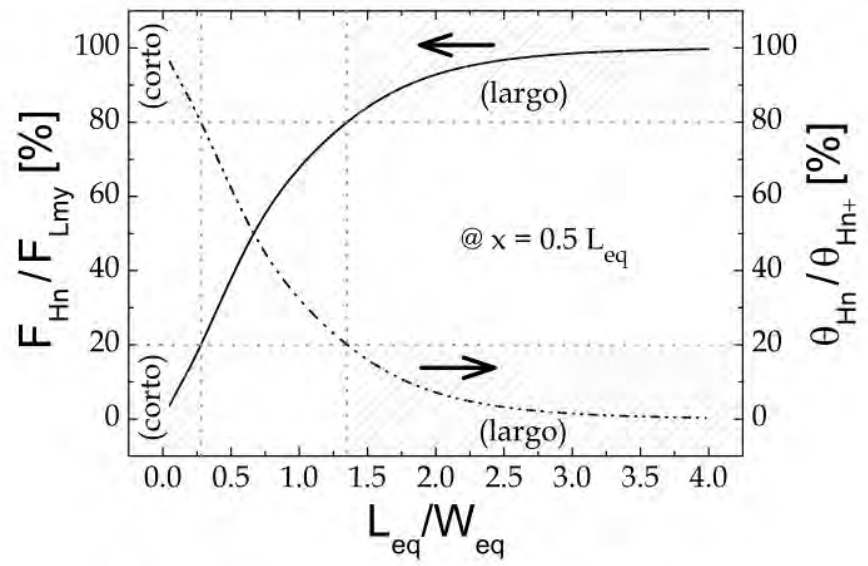

Figura 4: Razón entre fuerzas que actúan en la dirección de deflexión, $F_{H n} / F_{L m y}$, y razón entre el mínimo y el máximo valor del ángulo de Hall, $\theta_{H n} / \theta_{H n+}$.

Considerando los rangos propuestos en la Figura 4, un plato de Hall puede ser considerado largo cuando $L_{e q} / W_{e q} \geq 1.36$. En este caso, el efecto Hall es el dominante, porque el ángulo de Hall es reducido hasta valores por debajo del $20 \%$ de su valor máximo. Cuando $L_{e q} W_{e q} \leq 0.27$, el plato de Hall es corto manteniendo el ángulo de Hall entre el $80 \%$ y $100 \%$ de su valor máximo. En este caso, los efectos de deflexión de las líneas de corriente y de magnetorresistencia son dominantes [14, 15, 17]. En esta región es donde un SD-MAGFET debe ser diseñado para mejorar su sensibilidad.

\section{MACRO MODELO SPICE PROPUESTO}

La corriente de drenaje total de un SD-MAGFET puede ser aproximada como la corriente de drenaje de un MOSFET de canal rectangular con razón de aspecto $W_{e q} / L_{e q}$. La razón de aspecto rectangular equivalente, $W_{e q} L_{e q}$, para un SD-MAGFET puede ser estimada con la metodología propuesta en [16, 18]. En la aproximación de $W_{e q} / L_{e q}$ se usa un ángulo $\alpha$ que debe ser $\geq 75^{\circ}$. Entonces, la corriente generada por cada drenaje de un SD-MAGFET puede ser modelada por un MOSFET con razón de aspecto $0.5 \cdot W_{e q} / L_{e q}$ (ver Figura 5.(a)).

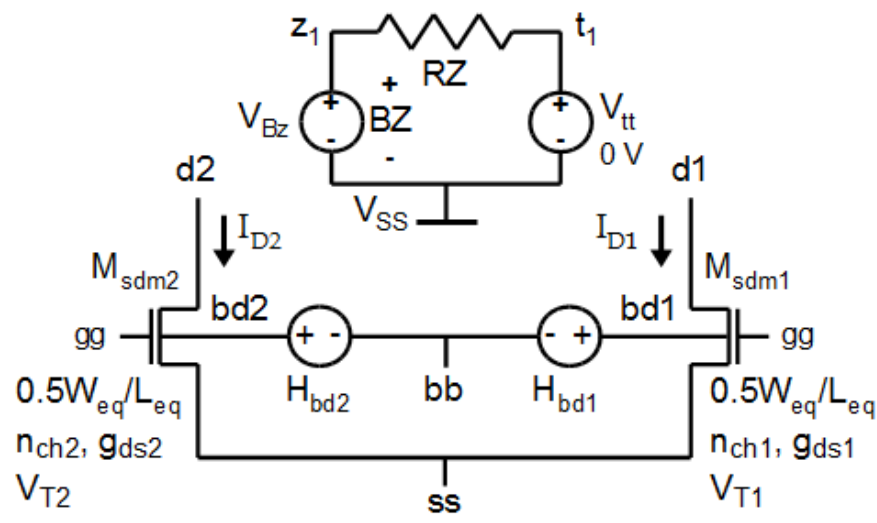

a) Descripción esquemática

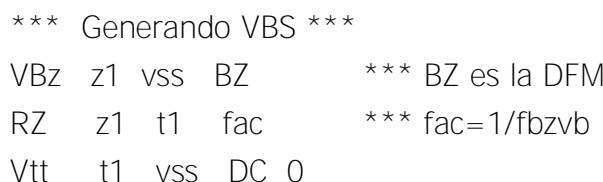

$\mathrm{Hbd} 2$ bd2 bb Vtt 1

Hbdl bd1 bb Vtt -1

*** SD-MAGFET $\quad * * *$

Msdm2 d2 gg ss bd2 MODN W=Wmm L=Leq

Msdm1 dl gg ss bd1 MODN W=Wmm L=Leq

b) Netlist SPICE

Figura 5: Macro modelo SPICE propuesto para un SDMAGFET.

La intensidad de la densidad de flujo magnético, $B_{z}$ normal al plano del canal, es introducida a través de una fuente de voltaje (ver Figura 5.(a)). $B_{z}$ genera una fuerza de Lorentz que actúa sobre cada portador de carga produciendo una diferencia de concentración de portadores entre las regiones del canal asociadas a cada drenaje. Esto puede ser modelado como una variación del voltaje substrato-fuente en cada drenaje, $V_{B S}$. Considerando que la variación de la corriente de drenaje de un MOSFET mantiene una apropiada linealidad con $V_{B S}$ [19] (ver Figura 6), es posible generar el desbalance entre las corrientes de drenaje variando $V_{B S}$ para cada drenaje. De esta 
ECIPERÚ

forma, este desbalance mantiene linealidad con $B_{z}$. Para cada drenaje, $V_{B S}$ tiene el mismo módulo pero signo opuesto, lo cual es especificado en las declaraciones de $H b d 1$ y $H b d 2$ en el netlist (ver Figura 5.(b)).

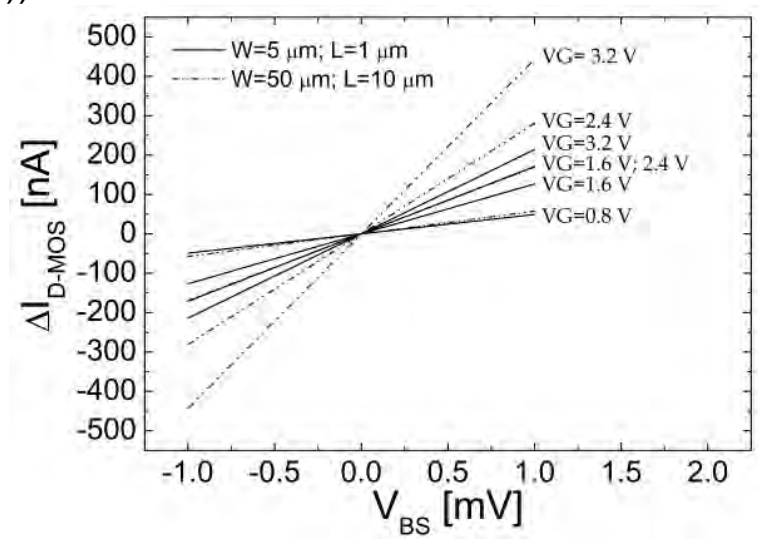

Figura 6 Variación de la corriente de drenaje de dos MOSFETs con $V_{B S}$ con respecto a su valor @ $V_{B S}=0 \mathrm{~V}$, para un voltaje drenaje-fuente de $3.2 \mathrm{~V}$.

Para generar el $V_{B S}$ apropiado para cada drenaje se usa el factor de conversión fbzvb, definido en la Ecuación (6). Este factor relaciona el desbalance entre las corrientes de drenaje de un SD-MAGFET debido a $B_{Z}, \Delta \boldsymbol{I}_{D}\left(B_{Z}\right)$, con la variación de corriente de un MOSFET de $W_{e q} / L_{e q}$ debido a un $V_{B S}$ del mismo valor numérico que $B_{Z}, \Delta I_{D M o s}\left(V_{B S}, W_{e q} / L_{e q}\right)$. Conociendo la sensibilidad del SD-MAGFET, $\Delta \boldsymbol{I}_{D}\left(B_{z}\right)$ puede ser estimado con la Ecuación (7), y $\Delta \boldsymbol{I}_{\boldsymbol{D}}$ $\operatorname{mos}\left(V_{B S}, W_{e q} / L_{e q}\right)$ a través de una simulación en SPICE. El netlist del macro modelo SPICE propuesto para un SD-MAGFET es mostrado en la Figura 5.(b), donde $W_{m m}=0.5^{*} W_{\text {eq }}$.

$$
\begin{gathered}
f b z v b=\frac{\Delta I_{D-M O S}\left(V_{B S} ; W_{e q} / L_{e q}\right)}{\Delta I_{D}\left(B_{z}\right)}=\frac{1}{R_{z}} \\
\Delta I_{D}\left(B_{z}\right)=\left|I_{D 2}-I_{D 1}\right|=S \cdot\left(I_{D 1}+I_{D 2}\right) \cdot B_{z}
\end{gathered}
$$

La forma propuesta para generar el desbalance entre las corrientes de drenaje crea niveles de inversión diferentes asociados a cada drenaje. Debido a esto, cada drenaje está asociado a una concentración de portadores $\left(n_{c h}\right)$, una conductancia $\left(g_{d s}\right)$ y un voltaje umbral $\left(V_{T}\right)$ diferente (ver Figura 5.(a)). Estos fenómenos físicos ocurren en un SD-MAGFET inmerso en una densidad de flujo magnético. El macro modelo propuesto permite incluir estos fenómenos físicos en una simulación SPICE, lo cual es un aporte sustancial respecto a otros macro modelos reportados en la literatura.

\section{RESULTADOS Y DISCUSIÓN}

Para las pruebas experimentales, fueron fabricados SD-MAGFETs en un chip con la tecnología CMOS $0.35 \mu \mathrm{m}$. En este trabajo, resultados obtenidos con un SD-MAGFET con $W=10 \mu m, L=2 \mu m, d=0.6 \mu m$, $u=0.4 \mu \mathrm{m}$ y $D S=0.5 \mu \mathrm{m}$ (ver Figuras 1 y 7 ) son presentados. Los nodos de fuente y substrato están cortocircuitados y son considerados como referencia $(0 \mathrm{~V})$. Voltajes de compuerta, $V_{G}$, y de drenaje, $V_{D}$, de $1.6 \mathrm{~V}, 2.4 \mathrm{~V}$ y $3.2 \mathrm{~V}$ fueron considerados. La densidad de flujo magnético, $\vec{B}$, fue generada con una corriente eléctrica $I_{S}$ fluyendo a través de una interconexión en metal 2 (ver Figuras 7). La intensidad de la componente de $\vec{B}$ normal al plano del canal, $\vec{B}_{Z}$, es aproximada usando la ley de Biot y Savart e integrando el aporte de cada sección diferencial en toda la sección transversal de la interconexión [14, 15 y 17]. Intensidades $90 \mu T, 180 \mu T$ y $275 \mu T$ fueron generadas en la superficie del canal para $\vec{B}_{Z}$.

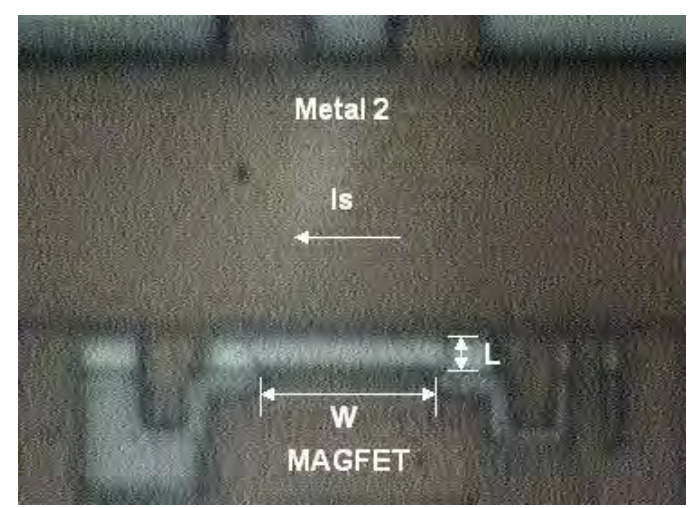

Figura 7 SD-MAGFET fabricado en la tecnología CMOS $0.35 \mu \mathrm{m}$.

Para cada valor de $V_{G}$ y $V_{D}$, la intensidad de $\vec{B}_{Z}$ fue variada en sentido positivo y negativo con un barrido de $I_{S}$. Cada punto del desbalance entre las corrientes de drenaje fue obtenido con un analizador de parámetros de semiconductores Agilent B1500A tomando 640 muestras. 10 conjuntos de puntos para cada barrido de densidad de flujo magnético fueron medidos. Luego se obtiene una regresión lineal y se elimina la componente de offset para obtener el desbalance real entre las corrientes de drenaje, $I_{D 2}-I_{D 1}$, (ver un ejemplo en la Figura 8.(a)). Todos los casos fueron en inversión fuerte.

\section{Verificación de Alta Sensibilidad}

Para las condiciones de polarización consideradas y usando la metodología experimental propuesta, las 
Figuras 8.(a), 8.(b) y 8.(c) muestran el desbalance entre las corrientes de drenaje medidas. Es notorio el incremento de $I_{D 2}-I_{D 1}$ cuando $V_{G}$ aumenta. Por otro lado, cuando el SD-MAGFET caracterizado pasa de la región lineal a la región de saturación, $I_{D 2}-I_{D 1}$ tiene un incremento significativo. Esto sucede porque la magnetorresistencia tiene mayor importancia en la región de saturación [11]. Sin embargo, para un mismo $V_{G}, I_{D 2}-I_{D 1}$ no es modificado apreciablemente por $V_{D}$.

Con los resultados mostrados en la Figura 8 y usando la Ecuación (1), la sensibilidad es estimada. Los resultados son mostrados en la Figura 9. En forma opuesta al desbalance entre las corrientes de drenaje, la sensibilidad aumenta notoriamente cuando $V_{G}$ disminuye (ver Figura 9.(a)). Esto ocurre porque $I_{D 2}-$ $I_{D 1}$ no se reduce tan rápidamente como la corriente total de drenaje cuando $V_{G}$ disminuye.

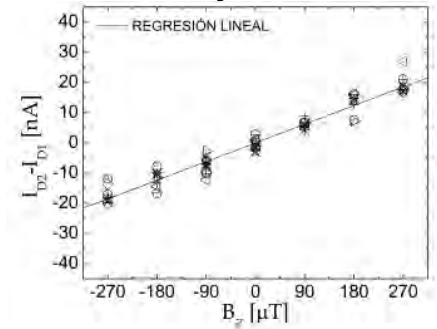

a) Muestras obtenidas a $V_{G}=1.6 \mathrm{~V}$ y $V_{D}=1.6 \mathrm{~V}$

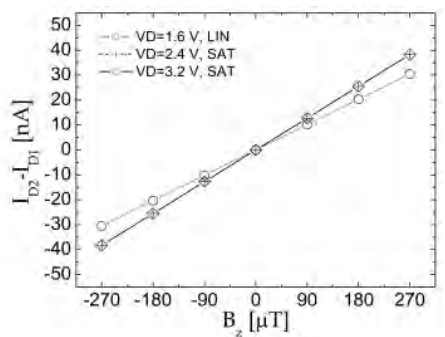

c) $V_{G}=2.4 \mathrm{~V}$

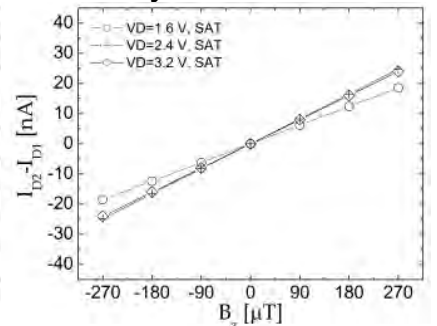

b) $V_{G}=1.6 \mathrm{~V}$

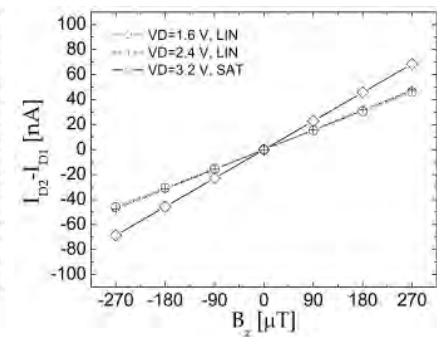

d) $V_{G}=3.2 \mathrm{~V}$
Figura 8: Desbalance entre las corrientes de drenaje para un SD-MAGFET con $W / L=10 \mu \mathrm{m} / 2 \mu \mathrm{m}$.

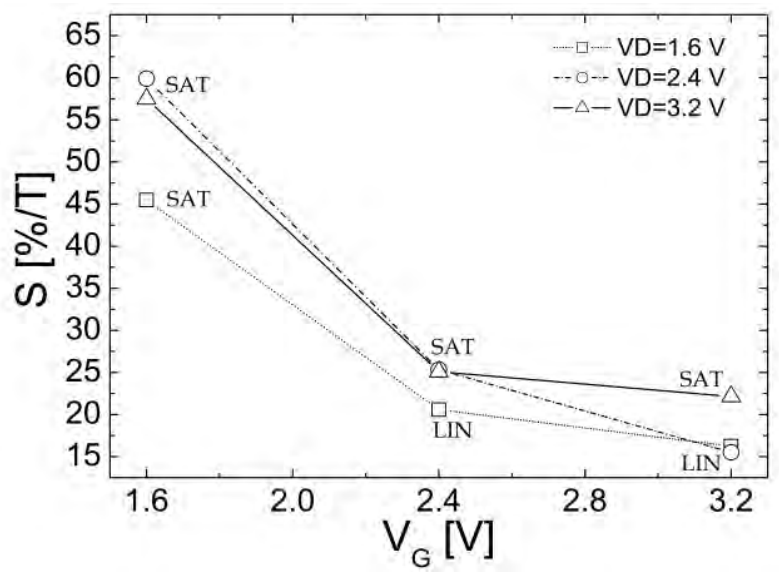

a) Variación con el voltaje de compuerta, $V_{G}$

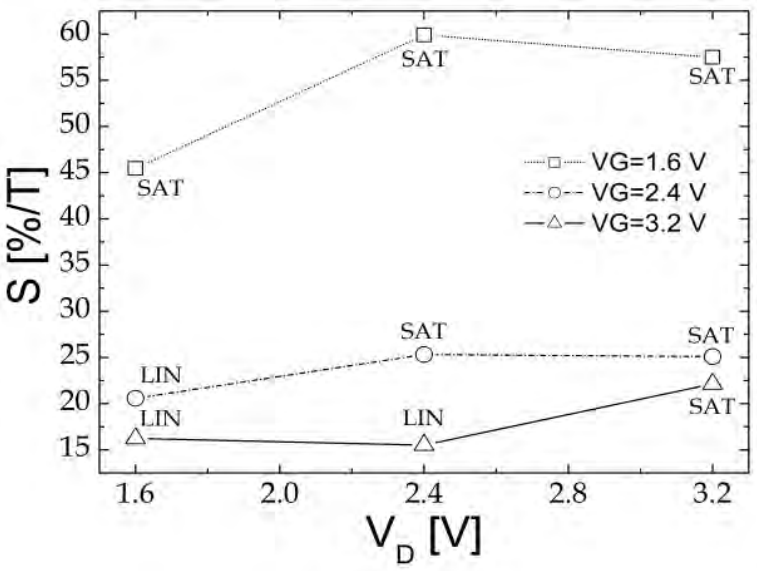

b) Variación con el voltaje de drenaje, $V_{D}$

Figura 9: Sensibilidad de un SD-MAGFET con $W=10 \mu m$ y $L=2 \mu m$ obtenida experimentalmente con diferentes condiciones de polarización.

Para un $V_{G}$ específico, por la misma razón indicada para $I_{D 2}-I_{D 1}$, la sensibilidad aumenta significativamente cuando el SD-MAGFET pasa de la región lineal a la región de saturación. De forma similar a $I_{D 2}-I_{D 1}$, la variación de la sensibilidad no es significativa con $V_{D}$ manteniéndose en una misma región de operación con el mismo $V_{G}$.

Validación del Macro Modelo SPICE Propuesto

Estimando apropiadamente la sensibilidad de un SDMAGFET $[4,15]$ usando el macro modelo SPICE propuesto, el desbalance entre las corrientes de drenaje puede ser obtenido con aceptable precisión. Esto es posible si el error en la estimación del factor fbzvb (ver Ecuación 6) es reducido. Para ello, la aproximación de la corriente total de drenaje generada por los MOSFETs (ver Figura 6) del macro modelo propuesto debe mantener un nivel de precisión aceptable.

Considerando el desbalance entre las corrientes de drenaje obtenido experimentalmente para un SDMAGFET de $W=10 \mu m$ y $L=2 \mu \mathrm{m}$, la Figura 10 muestra su aceptable reproducción usando el macro modelo SPICE propuesto. El error es $<1.6 \%$ para las condiciones de polarización consideradas. Por ello, el macro modelo SPICE propuesto es adecuado para simular SD-MAGFETs con una precisión aceptable.

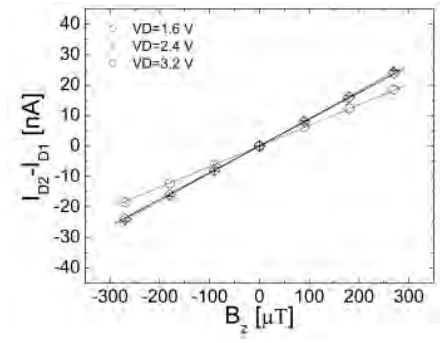

a) $V_{G}=1.6 \mathrm{~V}$

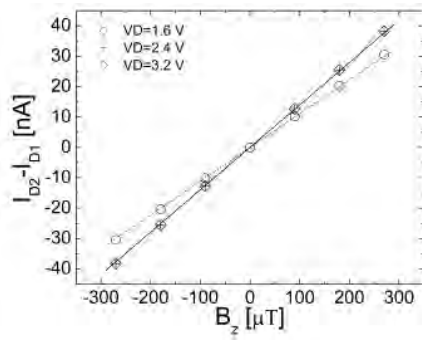

b) $V_{G}=2.4 \mathrm{~V}$ 


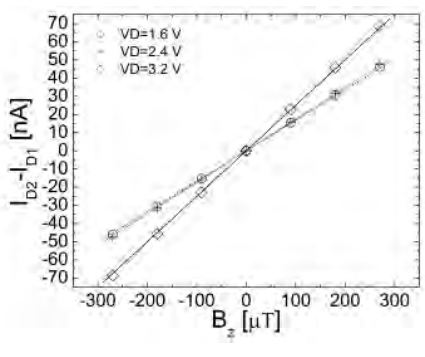

c) $V_{G}=3.2 \mathrm{~V}$

Figura 10: Desbalance entre las corrientes de drenaje obtenidas con el macro modelo SPICE propuesto (líneas continuas) y con mediciones experimentales (puntos discretos) para un SD-MAGFET con $W=10 \mu m$ y $L=2 \mu \mathrm{m}$.

\section{CRITERIOS DE DISEÑO PARA MEJORAR LA SENSIBILIDAD}

Sustentado en el análisis y los modelos propuestos y los resultados experimentales obtenidos, algunas precisiones para mejorar la sensibilidad de un SDMAGFET pueden ser indicadas. La razón $L_{e q} W_{e q}$ debe ser $\leq 0.27$, para que los efectos de deflexión de las líneas de corriente y de magnetorresistencia tengan mayor importancia. El voltaje compuertafuente debe ser reducido manteniendo el canal en inversión fuerte. El voltaje drenaje-fuente debe ser tal que asegure la operación en la región de saturación. Esto incrementa la importancia del efecto de magnetorresistencia en la sensibilidad. Considerando estos criterios, sensibilidades como las presentadas en este trabajo pueden ser obtenidas.

\section{CONCLUSIONES}

Los efectos galvanomagnéticos capaces de influir en la sensibilidad de un Split-Drain MAGFET (SDMAGFET) han sido analizados. Los modelos analíticos propuestos y validados experimentalmente, permiten establecer criterios de diseño apropiados para incrementar la sensibilidad de un SD-MAGFET. Con un SD-MAGFET con $L_{e q} / W_{e q}<0.27$, el ángulo de Hall se incrementa significativamente, con lo cual, los efectos de deflexión de las líneas de corriente y de magnetorresistencia llegan a ser importantes. Con esta condición, ambos efectos pueden lograr incrementar la sensibilidad significativamente. Por otro lado, la sensibilidad aumenta con la reducción del voltaje de compuerta y operando en la región de saturación. Con estos criterios, sensibilidades de hasta $59 \% / T$ fueron obtenidas experimentalmente con un SD-MAGFET con $W=10 \mu m$ y $L=2 \mu m$ en una tecnología CMOS de $0.35 \mu \mathrm{m}$. De forma complementaria, el macro modelo SPICE propuesto para un SD-MAGFET puede generar el desbalance entre las corrientes de drenaje con un error $<1.6 \%$. Por lo tanto, los resultados que han sido presentados muestran que es factible integrar SD-MAGFETs en una tecnología CMOS con un significativo incremento en la sensibilidad.

\section{AGRADECIMIENTOS}

Los autores agradecen al CONACyT de México por el apoyo parcial para la realización de este trabajo a través de la beca número 212028 y el proyecto número 83774-Y.

\section{REFERENCIAS}

[1] R. S. Popovic, Hall effect devices: magnetic sensors and characterization of semiconductors. Adam Hilger, 1991.

[2] H. P. Baltes y R. S. Popovic, "Integrated semiconductor magnetic field sensors," Proc. of the IEEE, vol. 74, no. 8, pp. 1107-1132, Aug. 1986.

[3] D. Killat, J. von Kluge, F. Umbach, W. Langheinrich y R. Schmitz, "Measurement and modeling of sensitivity and noise of MOS magnetic field-effect transistors," Sensors and Actuators A: Phys., vol. 61, no. 1-3, pp. 346-351, 1997.

[4] J. W. A. von Kluge et al., "An analytical model of MAGFET sensitivity including secondary effects using a continuous description of the geometric correction factor G," IEEE Trans. Electron Devices, vol. 46, no. 1, pp. 89-95, January, 1999.

[5] C. Rubio et al., "Modelling, design and test of a monolithic integrated magnetic sensor in a digital CMOS technology using a switched current interface system," Analog Integrated Circuits and Signal Processing, vol. 29, pp. 115-126, 2001.

[6] R. Rodríguez-Torres, E. Gutiérrez-Domínguez, R. Klima, y S. Selberherr, "Analysis of split-drain MAGFETs," IEEE Trans. Electron Devices, vol. 51, no. 12, pp. 2237-2245, Dec. 2004.

[7] Y. Yunruo, Z. Dazhong y G. Qing, "Sector splitdrain magnetic field-effect transistor based on standard CMOS technology," Sensors and Actuators A: Phys., vol. 121, no. 2, pp. 347-351, 2005.

[8] G. Qing, Z. Dazhong y Y. Yunruo, "CMOS magnetic sensor integrated circuit with sectorial MAGFET," Sensors and Actuators A: Phys., vol. 126, no. 1, pp. 154-158, 2006.

[9] Y. Sugiyama, H. Soga, M. Tacano y H. Baltes, "Highly sensitive split-contact magnetoresistor with AIAs/GaAs superlattice structures," IEEE Trans. Electron Dev., vol. 36, no. 9, pp. 1639-1643, 1989.

[10] J.W.A. von Kluge, "Analysis of split-current magnetic field sensitive resistors," Sensors and Actuators A: Phys., vol. 93, no. 2, pp. 103-108, 2001.

[11] V. N. Dobrovolsky y A. N. Krolevets, "Theory of magnetic-field-sensitive metal-oxide-semiconductor field-effect transistors," J. Appl. Phys., vol. 85, 
no. 3, 1956-1960, 1999.

[12] T. Pesic-Brdanin, N. Jankovic y D. Pantic, "SPICE MAGFET model and its application for simulation of magnetically controlled oscillator," Proc. 26th International Conference on Microelectronics, MIEL2008, pp. 503-506, 2008.

[13] S.-I. Liu et al., "SPICE macro model for MAGFET and its applications," IEEE Trans. Circuits Systems II, vol. 46, no. 4, pp. 370-375, Apr., 1999.

[14] Gerard F. Santillan-Quiñonez, V. Champac y R. S. Murphy, "A short-channel silicon-based split-drain MAGFET measuring from $90 \mu \mathrm{T}$," Proc. IEEE Latin American Symposium on Circuits and Systems, LASCAS'2010, pp. 108-111, Feb., 2010.

[15] Gerard F. Santillan Quiñonez, "Modeling and design of split-drain MAGFETs and possible applications in integrated circuit test", Disertación de Tesis de Doctorado por el Instituto Nacional de Astrofísica, Óptica y Electrónica, Julio, 2010.

[16] Gerard F. Santillan-Quiñonez, Roberto Murphy, y Victor Champac, "Equivalent rectangular active region and SPICE macro model for split-drain MAGFETs," Proc. 25th Symposium on Microelec- tronics Technology and Devices, SBMicro2010, ECS Transactions: Dielectric and Semiconductor Materials, Devices, and Processing, vol. 31, no. 1, pp. 393-400, September, 2010.

[17] Gerard F. Santillan-Quiñonez, Victor Champac y Roberto Murphy, "Exploiting magnetic sensing capabilities of Short Split-Drain MAGFETs," SolidState Electronics, Edit. Elsevier, vol. 54, no. 11, November, 2010, pp. 1239-1245.

[18] P. Grignoux y R. L. Geiger, "Modeling of MOS transistors with nonrectangular-gate geometries," IEEE Trans. Electron Devices, vol. ED-29, no. 8, pp. 1261-1269, Aug., 1982.

[19] Y. Tsividis, Operation and modeling of the MOS transistor, McGraw Hill, 1987.

E-mail: gsantil@ieee.org 